\title{
Aplicación del Big Data como estrategia de negocio en proyectos de emprendimiento en Ecuador
}

\section{Application of Big Data as a business strategy in entrepreneurship projects in Ecuador}

\author{
Ing. Mildred Verónica Merizalde Medina ${ }^{1}$ \\ mmerizalde@itsvr.edu.ec \\ Ing. Elizabeth Margoth Ballagán Guambo ${ }^{1}$ \\ eballagan@itsvr.edu.ec
}

Recibido: 1/12/2017, Aceptado: 1/02/2018

\begin{abstract}
RESUMEN
El presente artículo tiene como objetivo analizar la aplicación del Big Data en negocios de emprendimiento, diferenciar los términos de Big Data, Data Analytics y Data Mining, su relación y que deben considerar los emprendedores al momento de aplicarlos en sus modelos de negocios. Existen variedad de modelos relacionados al Big Data, estos se enfocan en procesos de negocio y marketing, los cuales fundamentan los criterios de decisión de una empresa. Se analizarán diferentes modelos, criterios relacionados al Big Data y estadísticas del emprendimiento en el Ecuador presentando como resultado, la consolidación de los principios que definen el almacenamiento y procesamiento de grandes volúmenes de información y la relación entre los factores críticos del emprendimiento, el ciclo de vida de los negocios emprendedores y los diferentes modelos Big Data que pueden aplicarse a lo largo del mismo.
\end{abstract}

Palabras clave: Big Data, Data Analytics, Data Mining, modelo de negocio, innovación, negocios emprendedores

\begin{abstract}
This paper analyzes the application of Big Data on entrepreneurial businesses, differentiates the terms Big Data, Data Analytics and Data Mining; their relationship and how entrepreneurs need to consider them before applying them to their business models. There are many Big Data models focused primarily on business and marketing processes given that these are the fundamentals a company uses to make their decisions. We analyze different models, criteria related to Big Data and statistics with entrepreneurs in Ecuador; presenting as a result the consolidation of best practices to store and process large amounts of data, the relationship between critical factors in the business, the lifecycle of a given business and the different Big Data models which can be applied thought the entire process.
\end{abstract}

\footnotetext{
${ }^{1}$ Docente del Instituto Tecnológico Vicente Rocafuerte
}

Revista científica Ciencia y Tecnología Vol 18 No 18 págs. 114-123

http://cienciaytecnologia.uteg.edu.ec 
Keywords: Big Data, Data Analytics, Data Mining, business model, innovation, entrepreneurial businesses

\section{Introducción}

Hablar de emprendimiento es hablar de innovación, la innovación no significa crear algo original; también significa reinventar, o también llamado, mejora de un producto o servicio. La innovación consiste en la implementación práctica de una idea en un nuevo producto o proceso; por lo tanto, juegan un papel muy importante la creatividad y las ideas.

\section{Desarrollo}

\section{Emprendimiento en el Ecuador}

En el Ecuador el emprendimiento ha tomado más fuerza en los últimos años, en el 2011, constaba ya entre los 15 países con mayor tendencia a iniciar negocios nuevos e innovadores. Para el 2015, en un estudio comparativo de la TEA entre países vecinos, Ecuador ocupaba un 33.6\%, seguido por Chile $25.9 \%$, Colombia $22.7 \%$ y Perú $22.2 \%$. Las estadísticas más representativas del Ecuador en cuanto a emprendimiento son:

Tabla 1: Índices TEA en Ecuador para los años 2015 y 2016

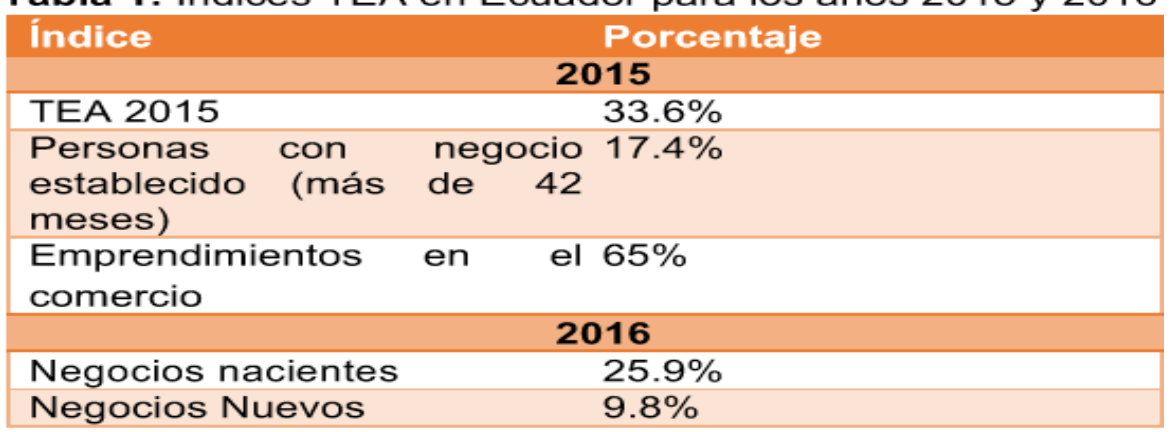

Fuente: Estudio GEM Ecuador 2015

Elaborado por: ESPAE-ESPOL 2016

Tabla 2: Emprendedores por nivel de educación

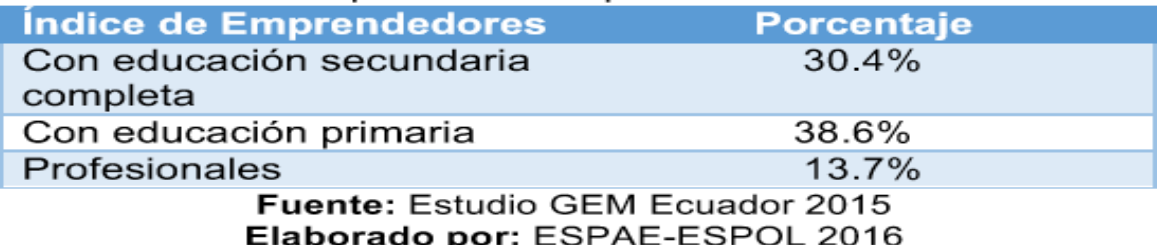

Tabla 3: Emprendedores por situación económica

\begin{tabular}{lc} 
Indice & Porcentajes \\
Emprendedores menores a & $45.1 \%$ \\
45 años auto-empleados & $17.6 \%$ \\
\hline $\begin{array}{l}\text { Emprendedores menores a } \\
45 \text { años con ingresos }\end{array}$ \\
familiares superiores a \$850 \\
\multicolumn{2}{c}{ Fuente: Estudio GEM Ecuador 2015 } \\
Elaborado por: ESPAE-ESPOL 2016
\end{tabular}




\section{Big data}

Big Data es considerado el siguiente nivel del Data Warehousing y el análisis de negocios, un fenómeno que crece aceleradamente y da lugar a la innovación y al cambio; su concepto no es nuevo, se ha ido madurando a lo largo de las dos últimas décadas gracias a la información colectada por diferentes fuentes.

Hablar de Big Data, es hablar de data multi-estructurada; aquella que se presenta en múltiples formatos y/o tipos y es generada por la interacción entre las personas y las máquinas, por medio de redes sociales o aplicaciones web (Arthur, 2013); su concepto se expande a la situación donde la logística de almacenamiento, el procesamiento y el análisis han sobrepasado los límites tradicionales de operación, por lo cual, las organizaciones han llegado a un punto en donde no saben cuanta data van a generar ni el cómo procesarla adecuadamente (EMC Education Services, 2015).

Los grandes volúmenes de información del Big Data se presentan en distintos niveles de complejidad, que no pueden ser procesados por herramientas comerciales 0 tecnologías comunes que pueden ser aplicadas a una base de datos tradicional; esta data proviene de sensores, equipos médicos, aeropuertos, redes sociales, datos de clientes en compañías de marketing, legales, de ventas, servicios, etc.

Considerar Big Data dentro de los procesos de negocio de una compañía sea esta grande, mediana o pequeña, constituye en un reto poco explorado hasta el momento; y surgen muchas interrogantes sobre cómo incluirlo de la manera más eficaz; o si ésta aportará en beneficios a los objetivos estratégicos; según Glass \& Callahan (2014), existen 11 principios que toda compañía debe considerar para introducir Big Data como parte de sus procesos:

- Enfocarse en el cliente.

- Contratar al personal apropiado, creativo y con habilidades analíticas. Mantener control sobre la tecnología.

- Definir métricas, métricas y más métricas.

- Mantenerse al frente de los procesos y los datos que los involucran.

- Realizar una auditoría de datos y esforzarse por integrar los silos de datos.

- Cooperar con los departamentos de tecnología, recursos humanos y otros usuarios interesados.

- Aplicar buenas prácticas de limpieza de datos.

- Desarrollar una estrategia, un camino a seguir, sin descuidar el deterioro de los datos.

Data Analytics

Data Analytics, busca definir el valor de vida útil del cliente (CVL), a partir de su análisis desde diferentes perspectivas. Como parte del Data Analytics, se consideran nuevos tipos de análisis de datos tales como:

Análisis Web. - Medición, colección, análisis y reporte de internet de los datos para propósitos de entendimiento y optimización de la web. (Definición de la Asociación de Análisis Digital, DAA) 


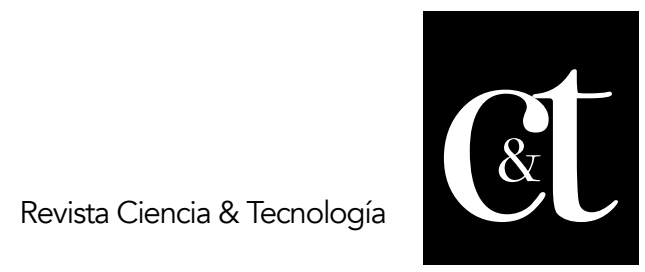

No. 18, 30 de abril de 2018

ISSN impreso: 1390 - 6321

Análisis de Redes Sociales. - Consiste en capturar información de los usuarios a través de sitios de redes sociales, permitiendo la personalización de la publicidad, ofertar servicios, definir presupuestos y medir impactos.

Big Data Analytics se forma de dos conceptos: el análisis predictivo y prescriptivo. El análisis predictivo, consiste en describir el futuro tomando en consideración información del pasado; esto se realiza con herramientas estadísticas tradicionales. El análisis prescriptivo, consiste en definir directrices futuras, actividades u obtener óptimos resultados evaluando la información pasada.

\section{Data Mining}

El Data Mining es un conjunto de técnicas aplicadas con el propósito de descubrir una estructura o definir un patrón, difiere de la estadística y los métodos tradicionales utilizados para el análisis de los datos y se está consolidando en un nuevo paradigma que, con la ayuda de estrategias y herramientas, busca maximizar el poder de la predicción por medio de modelados estadísticos (Attewel \& Monaghan, 2015).

Definir un modelo predictivo en base a algoritmos estadísticos, es el objetivo más común en la aplicación de esta técnica; existen muchos campos de desarrollo para data mining, tales como: bases de datos, ciencia, estadísticas, patrones de reconocimiento, neurociencia computacional, aprendizaje de computadores, inteligencia artificial, procesos de extracción de conocimiento (KDD)13.

Para asumir los retos de un proyecto en Data Mining, se deben considerar las siguientes fases (Attewel \& Monaghan, 2015):

Análisis de datos. - aunque sea un volumen de datos considerable, ésta debe ser analizada de una manera rápida por medio de la definición de pequeños casos, los mismos que nos presentarán patrones de conducta.

Construcción de variables. - consiste en definir variables o características que se convertirán en fuertes criterios de predicción o clasificación.

Selección de características. - Identificar las variables más representativas, aquellas que nos van a permitir resolver mayor número de problemas.

Extracción de las características. - consiste de tres procesos principales: el análisis de los componentes principales (PCA), análisis de los componentes independientes (ICA), combinaciones aleatorias y descomposición de los valores.

Construcción del modelo. - con los datos analizados y evaluados de las etapas anteriores, definir varios modelos, compararlos y seleccionar aquel que muestre mayor precisión de predicción.

Modelos relacionados al Big Data, Data Analytics y Data Mining

Con el nacimiento del Big Data, ya se analizan muchos modelos de aplicación con el objetivo de aprovechar al máximo la información generada: 
Modelo Big Data Marketing. - Consiste en el proceso de colectar, analizar y ejecutar los datos obtenidos para fomentar la participación del cliente, maximizar los resultados de marketing y poder obtener métricas que nos permitan llevar una contabilidad efectiva. La idea de este modelo es como utilizar el Big Data más eficiente (Arthur, 2013).

Modelo Data Analytics. - Consiste en definir el/los problemas a ser resueltos mediante análisis de los datos necesarios identificados y seleccionados para un fin específico. Toda la data debe ser compilada en un área o repositorio ya sea un datamart o un datawarehouse (Baesens, 2014).

Ciclo de vida de los Negocios de Emprendimiento. - Consiste en definir las fases operacionales de las empresas emprendedoras y las características de estas.

Modelo de Negocio de Big Data. - Consiste en definir los diferentes aspectos del negocio aplicando Big Data, desde la generación de la idea hasta la creación del producto y su valor. Procesos de Negocio Big Data. - Consisten en definir los modos de análisis, recolección e integración de los datos; los mismos que pueden ser personalizados y acoplarse a la diversidad de la información.

Modelo de Procesamiento de Datos. - Define la aplicación los procesos técnicos para Big Data, Data Analytics o Data Mining, dependiendo de la naturaleza y los requerimientos del negocio.

\section{Resultados}

En base al análisis de los puntos de vista estudiados, podemos observar que existen muchos criterios para determinar una definición completa del término Big Data, los autores concuerdan en la recolección de un gran volumen de datos que provienen de distintas fuentes, por lo cual no presentan una sola estructura, más bien se muestran en formatos diferentes.

Los autores, concuerdan en la definición del Big Data bajo tres principios fundamentales: Volumen, Velocidad, Variedad; sin embargo, existen algunos que los amplían, considerando Veracidad, Valor, Variabilidad y Visualización.

En cuanto a la veracidad, se considera la realidad de los datos, es decir que aquellos reflejen la situación o el aspecto que se está analizando; el valor, implica el nivel de influencia que ejerce la información proveniente del Big Data sobre una compañía, no solamente en cuanto al aspecto financiero, también en decisiones estratégicas; la visualización, implica las herramientas en las cuales la información es tratada y los resultados reflejados, de manera que los usuarios puedan entenderlos y definir estrategias en base a los mismos; por último, la variabilidad, consiste en el cambio continuo de estos resultados por efectos de nueva data obtenida o cambios de tendencias resultantes de los primeros análisis. 


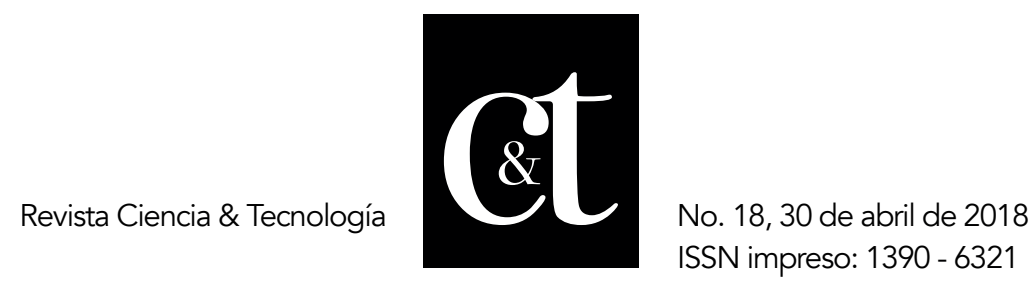

Tabla 4. Análisis de los principios del Big Data definidos por diferentes autores

\begin{tabular}{|c|c|c|c|c|c|c|c|c|}
\hline Autor & Año & Volumen & Velocidad & Variedad & Veracidad & Valor & Variabilidad & Visualización \\
\hline Lisa Arthur & 2013 & $x$ & $x$ & $\mathrm{x}$ & $x$ & $x$ & & \\
\hline $\begin{array}{l}\text { Krish } \\
\text { Krishnan }\end{array}$ & 2015 & $x$ & $x$ & $x$ & & & & \\
\hline $\begin{array}{l}\text { Fernando } \\
\text { Lafrate }\end{array}$ & 2015 & $x$ & $x$ & $x$ & & $x$ & & \\
\hline $\begin{array}{l}\text { Minelli, } \\
\text { Chambers, } \\
\text { Dhiraj }\end{array}$ & 2013 & $x$ & $x$ & $x$ & & & & \\
\hline $\begin{array}{l}\text { Frank } \\
\text { Ohlhorst }\end{array}$ & 2013 & $x$ & $x$ & $x$ & $x$ & & & \\
\hline $\begin{array}{l}\text { Mark Van } \\
\text { Rijmenam }\end{array}$ & 2014 & $x$ & $x$ & $x$ & $x$ & $x$ & $x$ & $x$ \\
\hline
\end{tabular}

Fuente: Criterios de diferentes autores y sus estudios sobre Big Data, Data Mining, Data Analytics

Elaborado por: los autores

Big Data, Data Analytics y Data Mining, no solo son considerados en aspectos técnicos; estos términos, son parte importante del negocio y su concepción, es por ello que muchos procesos relacionados al negocio y la generación del valor; son considerados como parte de las fases de análisis para la toma de decisiones estratégicas que permitan la creación de un proyecto o servicio exitoso y por supuesto, participan activamente a lo largo de la construcción de la empresa. Existen procesos para cada una de estas especialidades, para el emprendimiento y la administración, concepción y creación del negocio con el objetivo de lograr la madurez de una empresa. 


\section{Tabla 5. Definición de modelos de negocio, procesamiento de datos y} emprendimiento

\begin{tabular}{|c|c|c|c|}
\hline Autor & Ano & Modelo & Procesos \\
\hline \multicolumn{4}{|l|}{ Negocios } \\
\hline Bart Baesens & 2014 & $\begin{array}{l}\text { Procesos de Negocio } \\
\text { Big Data }\end{array}$ & $\begin{array}{l}\text { Procesos inteligentes } \\
\text { Procesos de descubrimiento } \\
\text { (mineria de datos y análisis) } \\
\text { Integración con Data Analytics. }\end{array}$ \\
\hline GEM & 2015 & $\begin{array}{l}\text { Modelo del ciclo de } \\
\text { vida de los negocios } \\
\text { emprendedores }\end{array}$ & 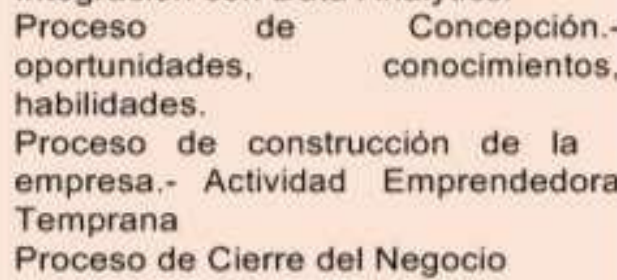 \\
\hline Bill Schmarzo & 2016 & $\begin{array}{l}\text { Modelo de Negocio } \\
\text { Big Data }\end{array}$ & $\begin{array}{l}\text { Monitoreo de Negocio } \\
\text { Definir Ideas de Negocio } \\
\text { Optimización del Negocio } \\
\text { Financiamiento del Negocio } \\
\text { Evolución del Negocio }\end{array}$ \\
\hline \multicolumn{4}{|l|}{ Marketing } \\
\hline Lisa Arthur & 2013 & Big Data Marketing & $\begin{array}{l}\text { Administración del Marketing } \\
\text { Integrado } \\
\text { Administración del Marketing de } \\
\text { Operaciones } \\
\text { Administración de la interacción del } \\
\text { cliente. } \\
\text { Mensajería Digital } \\
\text { Marketing Digital }\end{array}$ \\
\hline \multicolumn{4}{|c|}{ Procesamiento y análisis de datos } \\
\hline Krishnan Krish & 2015 & $\begin{array}{l}\text { Modelo } \\
\text { Procesamiento } \\
\text { Datos }\end{array}$ & $\begin{array}{l}\text { Colectar información } \\
\text { Procesar información } \\
\text { Administrar información } \\
\text { Generar información }\end{array}$ \\
\hline $\begin{array}{l}\text { Bart } \\
\text { Krish }\end{array}$ & 2014 & Data Analytics & $\begin{array}{l}\text { Definir la data a utilizar para la } \\
\text { aplicación. } \\
\text { Seleccionar la data } \\
\text { Procesar y limpiar la data. } \\
\text { Analizar la data } \\
\text { Interpretar y evaluar los resultados. }\end{array}$ \\
\hline
\end{tabular}

Fuente: Estudios del GEM 2015 Ecuador

Elaborado por: los autores

Analizando los diferentes modelos expuestos para áreas de negocios, marketing y procesamiento de datos, podemos observar que, para los casos de emprendimiento, la aplicación del Big Data puede ser aprovechada si se considera para áreas tales como: negocio y marketing. 


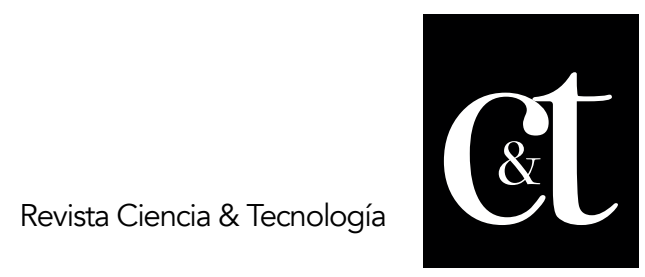

No. 18, 30 de abril de 2018

ISSN impreso: 1390 - 6321

En el área de negocios, se puede observar que el modelo de negocio Big Data, tiene mucha relación el ciclo de vida de los negocios emprendedores; es decir, puede convertirse en un factor clave para el éxito o fracaso de una compañía puesto que, durante sus primeras fases se pueden aplicar modelos de análisis con datos provenientes de redes sociales, estudios de mercado u otras compañías para determinar tendencias y lograr predecir si un producto puede o no lograr la acogida deseada.

En el área de marketing se puede determinar, en base a los cuadros anteriores, que los modelos de procesamiento de datos y marketing en Big Data guardan estrecha relación; puesto que, en todos ellos, se pueden permitir los cambios de estrategias a medida que las tendencias varíen, logrando que los emprendimientos se vayan edificando sobre pilares sólidos de conocimiento del mercado y su producto logre la evolución deseada.

Analizando los modelos planteados por los autores y el ciclo de vida de los emprendedores, obtenemos como resultado la aplicación de los diferentes modelos, por cada fase del Ciclo de Vida de los Negocios Emprendedores y la influencia de los factores críticos del emprendimiento presentada en el cuadro 1.

Los modelos de Negocio y Marketing en Big Data presentan mayor aplicación en las fases de concepción y construcción de la empresa, puesto que es vital un mayor esfuerzo en la definición y el monitoreo de las estrategias mientras la empresa se está solidificando hasta llegar a una posición un tanto estable en el mercado.

Los modelos de Data Analytics y Procesamiento de datos, se convierten en procesos netamente técnicos que involucran herramientas y el cómo los datos recolectados van a ser analizados, por ello vemos que su aplicación inicia desde finales de la concepción y presenta la mayor carga de esfuerzo en la etapa de construcción.

Los procesos de negocio son aquellos que van a aplicarse de forma constante a lo largo de las etapas del ciclo de vida, puesto que se convierten en un soporte a los modelos anteriores.

Por último, podemos observar que a lo largo del ciclo de vida de las empresas de emprendimiento existen factores críticos que ejercen influencias externas sobre las actividades internas de una compañía emprendedora; $y$, éstos pueden ejercer una influencia positiva o negativa, a lo largo de las diferentes fases de un negocio emprendedor. 


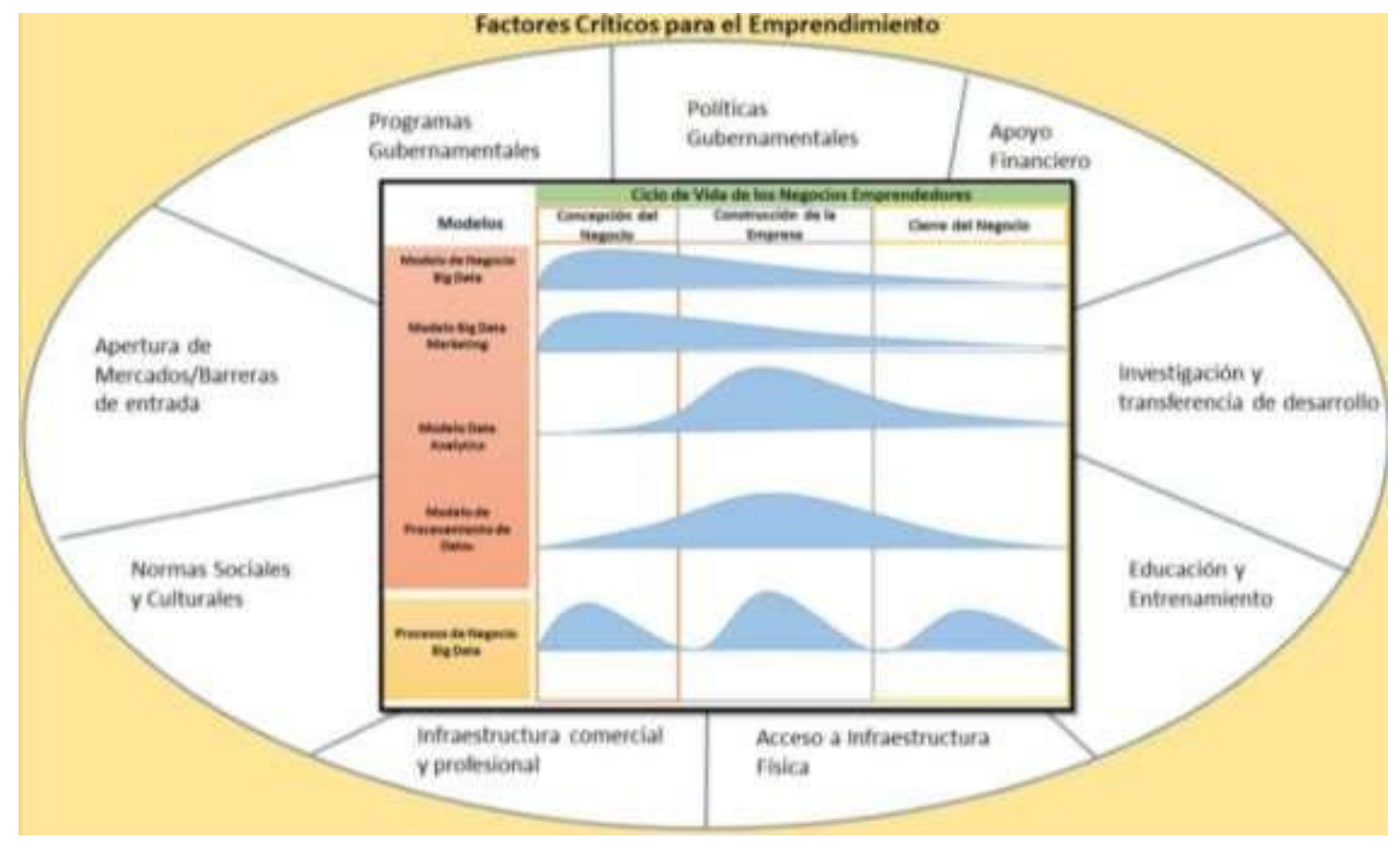

\section{Gráfico 1. Relación entre los factores críticos para el emprendimiento, el ciclo de vida de los negocios emprendedores y los modelos Big Data}

Fuente: Factores definidos en el estudio de Lasio, Caicedo, Ordeñana e Izquierdo (2015), criterios de diferentes autores y sus estudios sobre Big Data, Data Mining, Data Analysis e Innovación

Elaborado por: los autores de esta investigación

\section{Conclusiones}

En la actualidad Big Data, Data Analytics y Data Mining están muy relacionados a la innovación; con la creciente proliferación de las redes sociales, el internet y la digitalización de la información de las compañías, la evaluación de estrategias debe considerar muchos factores, índices, resultados y estudios generados en tiempo real, por lo cual la definición y ajuste de estas requiere un tiempo mínimo de respuesta. Los modelos de análisis de grandes volúmenes de información y las definiciones técnicas de cómo almacenarla nacen para satisfacer esa necesidad.

La información es el bien de valor en una empresa, y ésta puede servir como referencia para el nacimiento de nuevos productos y servicios; con ello se genera el emprendimiento. La aplicación de los modelos Big Data y el análisis de dicha información pueden influir positivamente en la consolidación de una empresa, puesto que, al utilizar estas técnicas, estamos asegurando la construcción de una estructura soportada en datos reales que permitirá tomar decisiones a tiempo en base a patrones definidos y tendencias obtenidas.

Existen muchos modelos que se relacionan al término Big Data, no necesariamente, éstos son técnicos, más bien, se están enfocando a ser partícipes de modelos y procesos de negocio y marketing puesto que su aplicación genera mayor valor 
agregado en esas disciplinas.

Los grandes volúmenes de información ya no son ideas del pasado, están aquí presentes y generándose diariamente; es decir, ya son una realidad para varias empresas; y, en Ecuador con mayor nivel de emprendimientos de la región puede llegar a ser una herramienta valiosa para analizar el mercado interno.

Es vital para el emprendedor, la educación y preparación en estas tecnologías nacientes para que pueda ser aplicarla de la forma óptima; por ello, no solo deben contar con el optimismo y el financiamiento de un nuevo negocio, sino con los conocimientos necesarios para tomar el mejor provecho de la información generada a su alrededor.

No debe ser mandatorio aplicar todos los procesos en las mismas proporciones, se debe buscar un balance entre los mismos y seleccionar aquellos que aporten significativamente al negocio emprendedor.

\section{Referencias bibliográficas}

Arthur, L. (2013). Big Data Marketing: Engage your customers more effectively and drive value. John Wiley \& Sons, Inc. New Jersey: Estados Unidos.

Attewell, P. \& Monaghan D. (2015). Data Mining for the social sciences. An introduction. University of California Press. California: Estados Unidos.

Baesens, B. (2014). Analytics in a Big Data World. The essential Guide to Data Science and its Applications. The Wiley \& SAS Business Series. Estados Unidos.

EMC Education Services (2015). Data Science and Big Data Analytics. Discovering, Analyzing, Visualizing and Presenting Data. John Wiley \& Sons, Inc. Indiana: Estados Unidos.

Glass, R. \& Callahan, S. (2014). The Big Data-Driven Business. How To use Big Data to win Customers, Beat competitors and Boost Profits. John Wiley \& Sons, Inc. New Jersey: Estados Unidos.

Lasio, V., Caicedo, G., Ordeñana, X. e Izquierdo, E. (2016). GEM Ecuador Report 2015. Espae-Espol. Ecuador. Disponible en: http://www.eltelegrafo.com.ec/especiales/Documentos/GEM-2015Ecuador.pdf 